\title{
Automatic Adaptive Space Segmentation for Reinforcement Learning
}

\author{
Yuki Komori, Akira Notsu*, Katsuhiro Honda, and Hidetomo Ichihashi \\ Department of Computer Sciences and Intelligent Systems, Graduate School of Engineering, \\ Osaka Prefecture University, Osaka, 599-8531, JAPAN
}

\begin{abstract}
We tested a single pendulum simulation and observed the influence of several situation space segmentation types in reinforcement learning processes in order to propose a new adaptive automation for situation space segmentation. Its segmentation is performed by the Contraction Algorithm and the Cell Division Approach. Also, its automation is performed by "entropy," which is defined on action values' distributions. Simulation results were shown to demonstrate the influence and adaptability of the proposed method.
\end{abstract}

Keywords : Reinforcement learning, Space segmentation, Entropy.

\section{Introduction}

Reinforcement learning is a framework for a strategy to learn through trial and error according to rewards provided by the environment [1]. In reinforcement learning, if the purpose of study is set, the agent automatically determines the best way to achieve the goal.

Q-learning [2], [3] is a typical reinforced study method that targets discrete states and actions. Solving a problem that has a continuous state and a continuous action requires discrimination of the continuous state and continuous action. We introduced the Contraction Method [5], and the Segmentation and Integration Method [6], into Learning Residual Entropy [7]. The Contraction Method contracts a state. The Segmentation and Integration Method segments a state and integrates two adjacent states. Learning Residual Entropy shows the degree of actions' converging.

In this paper, we investigated these methods. We considered how to divide in order to find the optimum solution faster, the technique by which an agent can acquire the best approach to automatically divide states, and the headstand stabilization problem of the pendulum.

\section{Q-learning}

Q-learning is a well-known reinforcement learning technique that works by learning an action-value function that gives the expected utility of taking given action $a$ in given state $s$ and following a fixed policy thereafter. One advantage of Q-

Manuscript received Feb. 28, 2012; revised Mar. 5, 2012; accepted Mar. 12, 2012

*Corresponding author: Akira Notsu (Phone: +81-72-254-9355, fax: +81-72-254-9915, E-mail: notsu@cs.osakafu-u.ac.jp)

(C) The Korean Institute of Intelligent Systems. All rights reserved.

It is the extended version of a paper awarded as a best presentation in ISIS2011. learning is that it can compare the expected utility $Q$ of available actions without requiring a model of the environment (usually, the agent selects the action with the maximum expected utility). Expected utility $Q$ is updated iteratively. For each state $s$ from state set $S$, and for each action $a$ from action set $A$, we update Q-value $Q$ that depends on current state $s$ of the agent, action $a$ selected by the agent, the next state $s$ ' of the agent after taking action $a$, and reward $r$ received by the agent after taking the action. An update is calculated by the following expression:

$$
Q(s, a) \leftarrow Q(s, a)+\alpha\left[r+\gamma \max _{a^{\prime} \in A\left(s^{\prime}\right)} Q\left(s^{\prime}, a^{\prime}\right)-Q(s, a)\right],(1)
$$

where $\alpha$ is the learning rate and $\gamma$ is the discount factor.

Once Q-learning has finished, the optimal policy and optimal value function have been found without continuously updating the policy during learning.

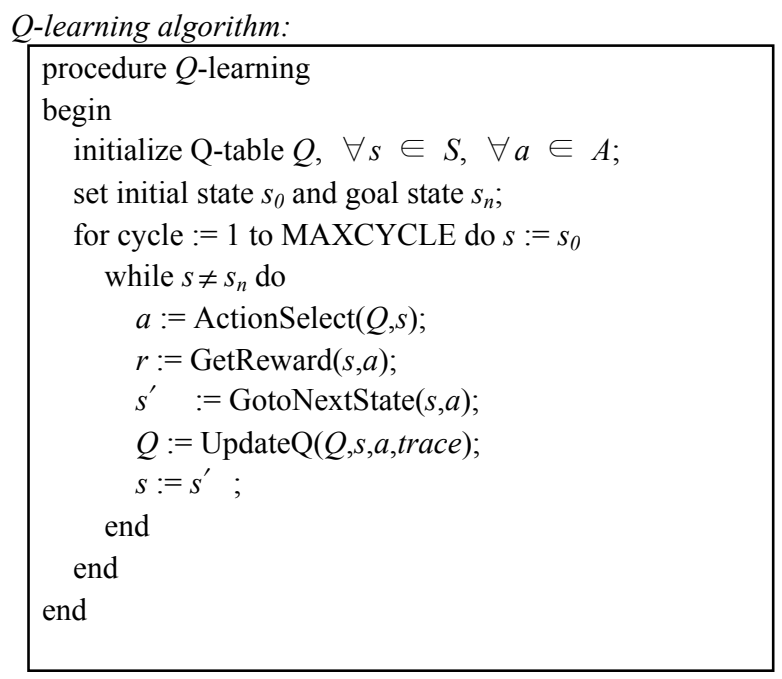

\section{Learning Residual Entropy}

The timing of the change of how to divide state space 
influences long-term reward and the speed of learning. However, finding the appropriate timing is difficult. Therefore, "Learning residual entropy" was proposed [7]. It expresses the grade of learning processes. For each state $S$, learning residual entropy $I(S)$ is defined as follows from action choice probability $p(S, a)$.

$$
I(S)=-\frac{1}{\log |a|} \sum_{a} p(S, a) \log (p(S, a)),
$$

where $p(S, a)$ is a probability of the internal state $S$ and action $a$. Now, $|a|$ is a number of possible actions. $I(S)$ is an index of to what degree uncertainty of actions remains at each state. In an unlearned case, $I(S)=\log |a| / \log |a|=1$. In an advanced learning case, particular action tends to be chosen in some cases. Then the more learning processes there are, the more $I(S)$ approaches 0 .

In addition, the average of $I(S)$ in every state which is included in episode $E$ at every episode and is called "Average of learning residual entropy" $I$, is as follows:

$$
I=\frac{1}{|E|} \sum_{S \in E} I(S)
$$

Now, $|E|$ is a number of states included in episode $E$. By using the learning residual entropy, there is no guarantee of always getting appropriate timing. However, this can be obtained with only information from the environment, so this can become an extremely powerful index.

\section{Single Pendulum Simulation}

We simulated the single pendulum standing task (Figure 1). This physical calculation is performed by the RungeKutta $4^{\text {th }}$ order method.

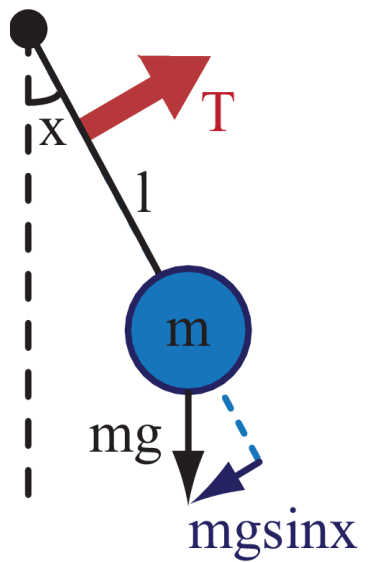

Fig. 1. Single pendulum

The following motion equation is simulated in increme nts of 0.05 seconds [4]:

$$
m \ddot{x}=-m g l \sin x-k \dot{x}+T
$$

where the parameters and variables are defined as follows:

$$
\begin{aligned}
& m=1(\mathrm{~kg}) \\
& l=1(\mathrm{~m})
\end{aligned}
$$

$k=0$

$g=9.8\left(\mathrm{~m} / \mathrm{s}^{2}\right)$

$-\pi<x \leq \pi$

$-3 \pi<\dot{x} \leq 3 \pi$

$-20 \leq T \leq 20$

1 iteration $=20$ seconds

1 learning cycle $=0.2$ seconds

$x_{0}=\pi, \dot{x}_{0}=0$ in the initial state

reward $r_{t}=\frac{|x|}{\pi}-0.5 \frac{|T|}{20}$

learning rate $\alpha=0.25$

discount factor $\gamma=0.9$

temperature $\tau=1$ at the first learning cycle, $\tau=0.001$

at the last learning cycle (linearly decreasing)

1000 episodes $\times 100$

angle $x$ and angular velocity $\dot{x}$ are segmented int

o 10 segments

torque $T$ is segmented into six segments

$x$ is segmented evenly, in the initial state

$\dot{x}$ and $T$ are segmented evenly

contraction ratio is 0.05 .

\subsection{Contraction Method}

We investigated an automatic contraction method through simulation [5].

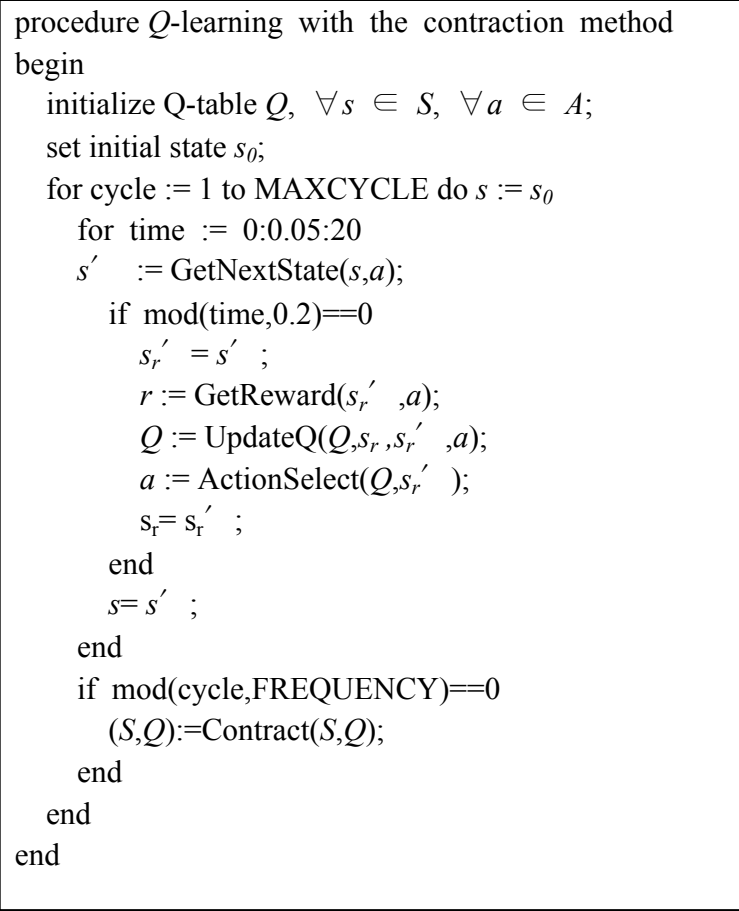

In this method, the space is initially evenly segmented, and contraction is performed at regular cycles by the Contract $(S, Q)$ functions. The state in which the visited frequency is the most is contracted by the $\operatorname{Contract}(S, Q)$ function. Then, other states are expanded with the same ratio as before. Expected utilities are undisturbed. Each various episode was contracted. These 
average rewards from 951 episodes to 1000 episodes are shown in TABLE I.

TABLE I

Frequency of contraction and average reward (average of 100 trials)

\begin{tabular}{c|c|c}
\hline $\begin{array}{c}\text { Frequency of } \\
\text { contraction }\end{array}$ & $\begin{array}{c}\text { Average } \\
\text { reward }\end{array}$ & $\begin{array}{c}\text { Contraction } \\
\text { count }\end{array}$ \\
\hline \hline every 5 episodes & 54.6862 & 200 \\
\hline every 10 episodes & 74.8555 & 100 \\
\hline every 15 episodes & 76.5142 & 66 \\
\hline every 20 episodes & $\mathbf{7 6 . 9 3 1 5}$ & $\mathbf{5 0}$ \\
\hline every 25 episodes & 76.1738 & 40 \\
\hline every 30 episodes & 75.3698 & 33 \\
\hline no contraction & 73.2671 & 0 \\
\hline
\end{tabular}

As a result, "every 20 episodes" had the best reward.

\subsection{Segmentation and Integration Method}

We investigated an automatic segmentation and integration method through simulation[6].

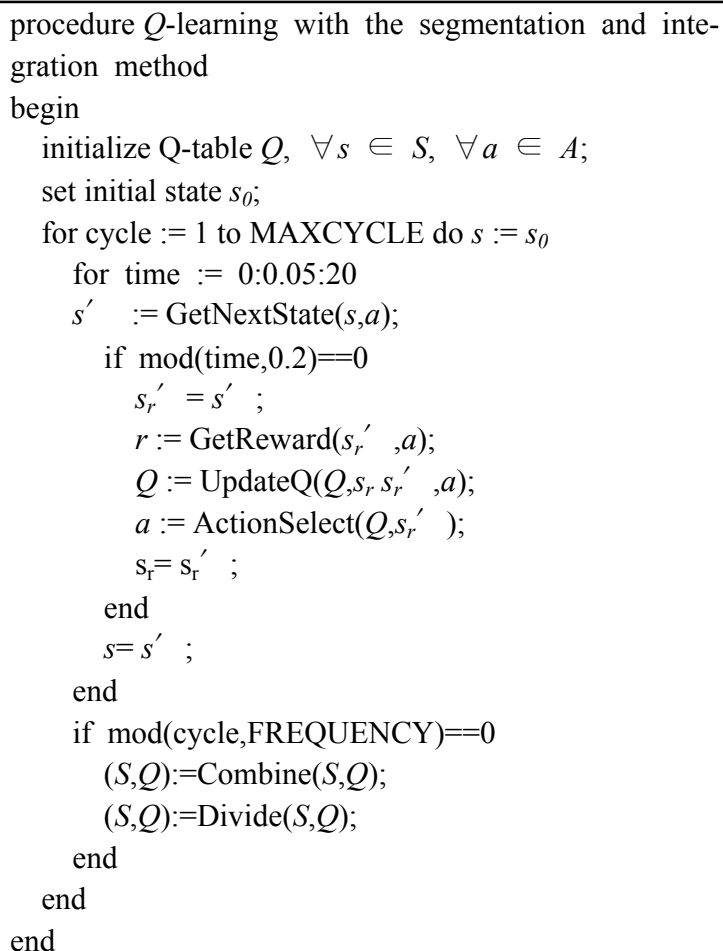

In this method, the space is initially evenly segmented, and segmentation and integration are performed at regular cycles by the Combine $(S, Q)$ and $\operatorname{Divide}(S, Q)$ functions. The neighboring states in which an average of the visited frequency is the least are integrated into a single state that has an average expected utility of the states by the Combine $(S, Q)$ function. The state in which the visited frequency is the most is equally segmented into two states that have the same expected utility by the $\operatorname{Divide}(S, Q)$ function. Other expected utilities are undisturbed.

In each various episode, the states were combined and divided. These average rewards from 951 episodes to 1000 episodes are shown in TABLE II.

TABLE II

Frequency of combining and dividing and the average reward (average of 100 trials)

\begin{tabular}{c|c|c}
\hline $\begin{array}{c}\text { Frequency of combining } \\
\text { and dividing }\end{array}$ & $\begin{array}{c}\text { Average } \\
\text { reward }\end{array}$ & $\begin{array}{c}\text { Contraction } \\
\text { count }\end{array}$ \\
\hline \hline every 50 episodes & 74.0592 & 20 \\
\hline every 100 episodes & 77.1982 & 10 \\
\hline every 200 episodes & $\mathbf{7 8 . 5 9 2 0}$ & $\mathbf{5}$ \\
\hline every 300 episodes & 76.2746 & 3 \\
\hline every 400 episodes & 75.6055 & 2 \\
\hline every 500 episodes & 74.6342 & 2 \\
\hline no combining and dividing & 73.2671 & 0 \\
\hline
\end{tabular}

As a result, "every 200 episodes" had the best reward.

4.3 Comparison of "Contraction method" and the "Segmentation and Integration Method"

Learning curves of the "Contraction Method" and "Segmentation and Integration Method" are shown in Figure 2.

This figure shows that the two methods got better reward than no-change states. In the "Segmentation and Integration Method," the reward was increasing especially every 200 episodes.

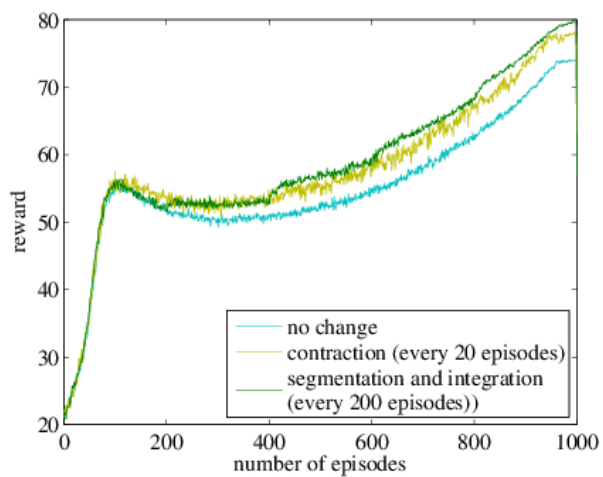

Fig. 2. Comparison of these methods (average of 100 trials)

\subsection{Learning Residual Entropy Approach}

We investigated an automatic learning residual entropy approach with the "Contraction Method" or "Segmentation and Integration Method" through simulation.

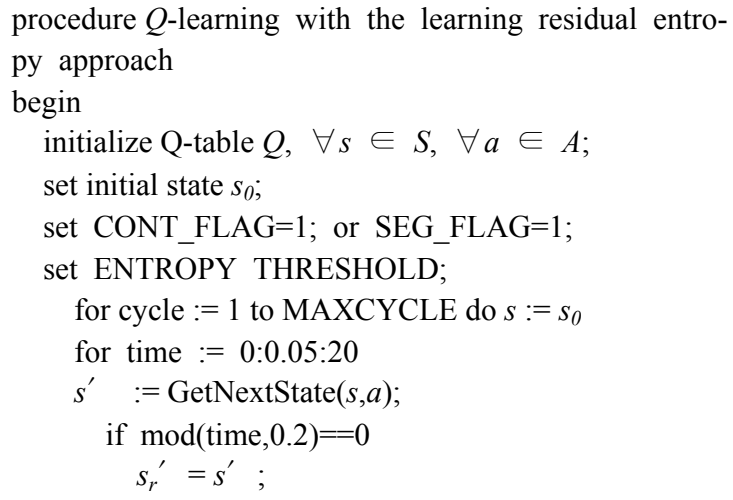

procedure $Q$-learning with the learning residual entro- 


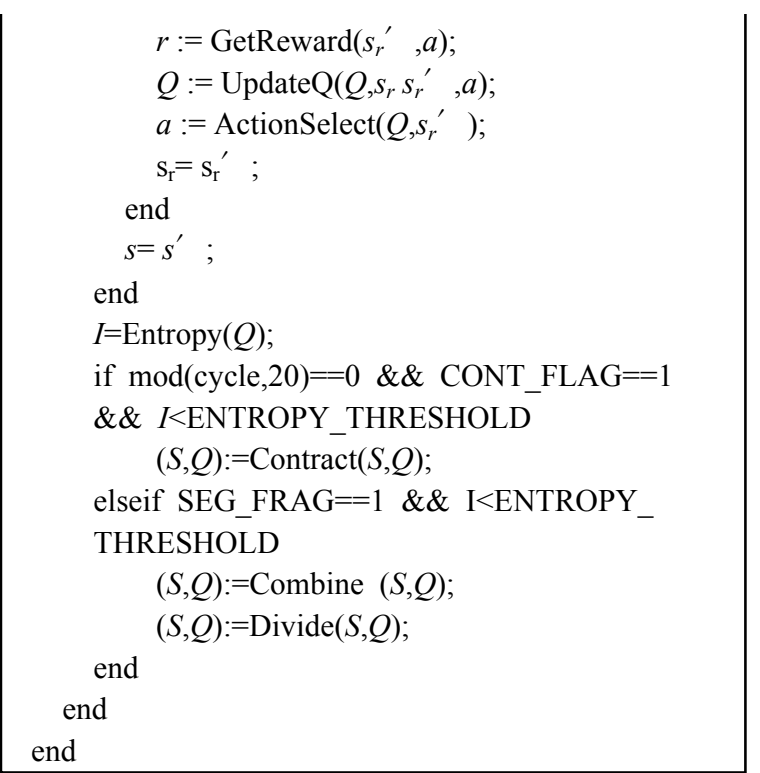

In this method, the "Contraction Method" or "Segmentation and Integration Method" is done when learning residual entropy is under the entropy threshold. However, in the "Contraction Method," the average entropy of the previous 20 episodes is used because contractions make little change in action values' distributions; namely, learning residual entropy changes little. In the "Segmentation and Integration Method," the entropy of the previous episode is used. These average rewards from 951 episodes to 1000 episodes are shown in TABLES III and IV, and the entropy method's rewards are shown in Figure 3. Average entropy and an example of entropy are also shown in Figure 4 and 5.

TABLE III

Entropy approach with "Contraction Method" (average of 100 trials)

\begin{tabular}{c|c|c}
\hline Entropy threshold & Average reward & Contraction count \\
\hline \hline 0.3 & 73.4586 & 0 \\
\hline 0.6 & 75.4468 & 34.82 \\
\hline 0.65 & 76.1829 & 40.69 \\
\hline $\mathbf{0 . 7}$ & $\mathbf{7 7 . 1 4 8}$ & $\mathbf{4 3 . 9 5}$ \\
\hline 0.75 & 76.2985 & 44.74 \\
\hline 0.8 & 76.8721 & 45.98 \\
\hline 0.9 & 76.7689 & 46.94 \\
\hline more than 1.0 & -- & 50 \\
\hline \multicolumn{2}{|c}{}
\end{tabular}

TABLE IV

Entropy approach with "Segmentation and Integration Method" (average of 100 trials)

\begin{tabular}{c|c|c}
\hline Entropy threshold & Average reward & Contraction count \\
\hline \hline 0.3 & 73.3312 & 0 \\
\hline 0.5 & 74.0798 & 0.69 \\
\hline 0.5 & 75.971 & 2.47 \\
\hline $\mathbf{0 . 6}$ & $\mathbf{7 7 . 1 7 0 7}$ & $\mathbf{9 . 8 6}$ \\
\hline 0.65 & 73.5105 & 38.92 \\
\hline 0.7 & 67.3112 & 127.66 \\
\hline 0.9 & 58.5275 & 940.15 \\
\hline
\end{tabular}

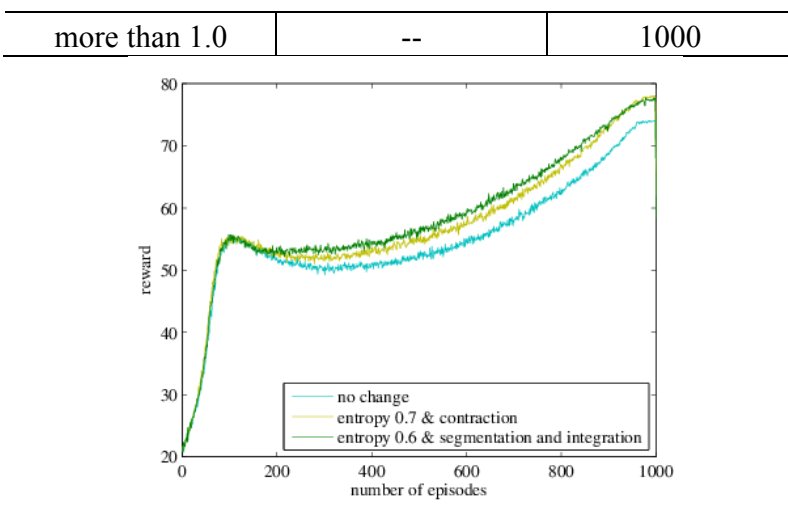

Fig. 3. Entropy methods reward (average of 100 trials)

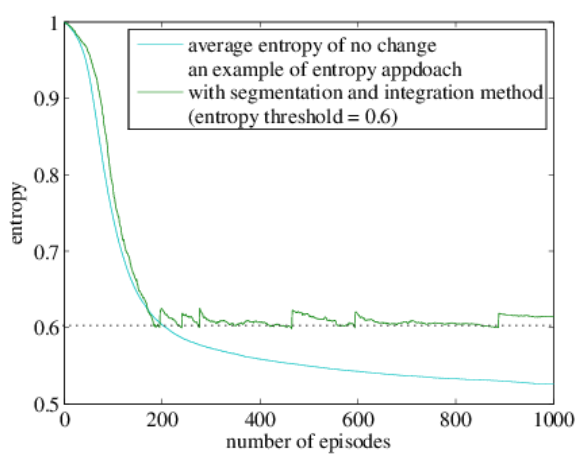

Fig. 4. Entropy example with "conventional approach" and "Segmentation and Integration Method"

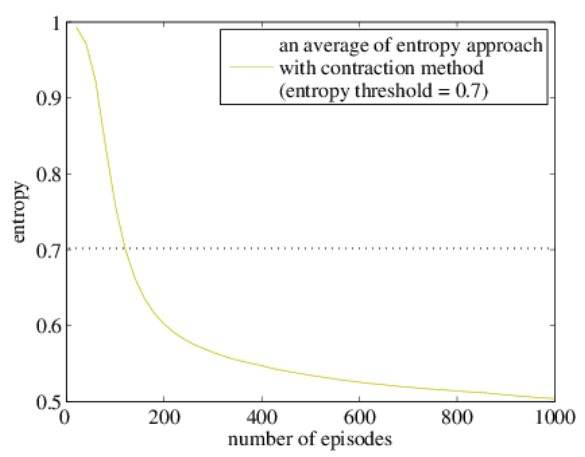

Fig. 5. Entropy example with "Contraction Method"

In the Contraction Method, contraction every 20 episodes was the best. In the Segmentation and Integration Method, segmentation and integration every 200 episodes was the best. Both methods could get better rewards than the case of evenly segmented states. In the Segmentation and Integration Method, the reward increased in particular for every episode of changing how to divide state space, which indicates that the method can be used to determine an appropriate segmentation method automatically by time. In the learning residual entropy approach with the Segmentation and Integration Method, states were segmented and integrated after approximately converging. Then, learning residual entropy increased, and after waiting for new converging, the next segmentation and integration were 
done. Therefore, the first objective, which is to find good timing for changing state space, was accomplished.

However, in the learning residual entropy approach with the contraction method, learning residual entropy was not very increased after contraction, so we took an average of 20 episodes. From this, in the early stages of learning (about the first 100 episodes), contraction was not done and after approximately converging, contraction was done at fixed intervals. Thus, this method can also find good timing for changing state space.

\section{Conclusions}

Q-learning is a typical reinforced study method that targets discrete states and actions. However, finding appropriate space segmentation types is difficult. In this paper, we investigated the "Contraction Method," "Segmentation and Integration Method," and "Learning Residual Entropy Approach" with these two methods through single pendulum simulations. We showed changing space segmentation types while learning was effective and learning residual entropy could find good timing for changing state space.

Finding the appropriate entropy threshold automatically or finding more effective method to find good timing for changing state space remains as the future work.

\section{Acknowledgment}

This work was supported in part by the Ministry of Education, Culture, Science and Technology, Japan under Grant-in-Aid for Scientific Research No. 21700242 and funded by the Ministry of Internal Affairs and Communications, Strategic Information and Communications R\&D Promotion Program.

\section{References}

[1] R. S. Sutton, "Learning to Predict by Method of Temporal Differences," Machine Learning, vol. 3, 1, pp. 9-44, 1988.

[2] T. Jaakkola, M. Jordan, and S. P. Singh, "On the Convergence of Stochastic Iterative Dynamic Programming Algorithms," Neural Computation, vol. 6, pp. 341-362, 1992.

[3] C. J. C. H. Watkins and P. Dayan, "Technical Note: QLearning," Machine Learning, vol. 8, pp. 56-68, 1992.

[4] Y. Kashimura, A. Ueno, and S. Tatsumi, "A Continuous Action Space Representation by Particle Filter for Reinforcement Learning," JSAI2008, pp. 118-121, 2008.

[5] A. Notsu, H. Honda, H. Ichihashi, and H. Wada, "Contraction Algorithm in State and Action space for Qlearning," Proc. of SCIS\&ISIS, pp. 93-96, 2009.

[6] A. Notsu, H. Wada, H. Honda, and H. Ichihashi, "Cell Division Approach for Search Space in Reinforcement
Learning," International Journal of Computer Science and Network Security, vol. 8, no. 6, 2008.

[7] A. Ito and M. Kanabuchi, "Speeding up Multi-Agent Reinforcement Learning by Coarse-Graining of Perception Hunter Game as an Example," IEICE Trans. D, vol. J84-D1, no. 3, pp. 285-293, 2001.

[8] M. Nagayoshi, H. Muraoand, and H. Tamaki, "Switching Reinforcement Learning to Mimic an Infant's Motor Development Application to Two-dimensional Continuous Action Space," Proc. SICE Annual Conference 2010 (SICE 2010), pp.243-246 (TA09-3(on DVD-ROM)), 2010.

Yuki Komori received the B.Eng. degree from Osaka

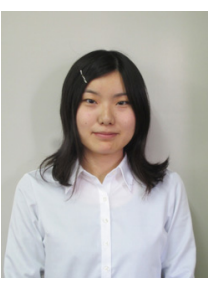
Prefecture University in 2011.

She is with the Graduate School of Engineering, Osaka Prefecture University. Her research interests include reinforcement learning and multi-agent simulation.

E-mail: komori@hi.cs.osakafu-u.ac.jp

Akira Notsu received the B.E., M.I. and D. Informatics

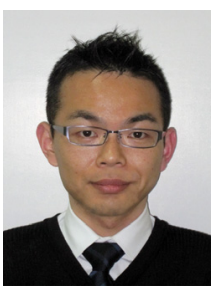
degrees from Kyoto University in 2000, 2002 , and 2005, respectively.

$\mathrm{He}$ is currently an Assistant Professor, Department of Computer Science and Intelligent Systems, Osaka Prefecture University. His research interests include agent-based social simulation, communication networks, game theory, human machine interface, and cognitive engineering.

E-mail: notsu@cs.osakafu-u.ac.jp

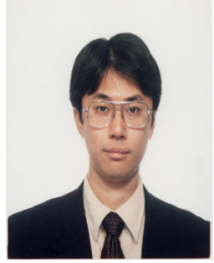

Katsuhiro Honda (M'01) received the B.E., M.E. and D.Eng. degrees in industrial engineering from Osaka Prefecture University, Osaka, Japan, in 1997, 1999, and 2004, respectively.

$\mathrm{He}$ is currently an Assistant Professor, Department of Computer Science and Intelligent Systems, Osaka Prefecture University. His research interests include hybrid techniques of fuzzy clustering and multivariate analysis, data mining with fuzzy data analysis, and neural networks. He received a paper award and a young investigator award from the Japan Society for Fuzzy Theory and Intelligent Informatics (SOFT) in 2002 and 2005, respectively, and gave a tutorial titled "Introduction to Clustering Techniques" at the 2004 IEEE Int. Conf. on Fuzzy Systems (FUZZ-IEEE 2004).

E-mail: Honda@cs.osakafu-u.ac.jp 


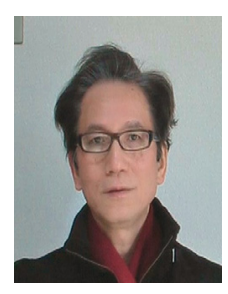

Hidetomo Ichihashi (M'94) received the B.E. and D.Eng. degrees in industrial engineering from Osaka Prefecture University, Osaka, Japan, in 1971 and 1986, respectively.

From 1971 to 1981, he was with the Information System Center of Matsushita Electric Industrial Co., Ltd., Tokyo, Japan.
From 1981 to 1993, he was a Research Associate, Assistant Professor, and Associate Professor at Osaka Prefecture University, where he is currently a Professor in the Department of Computer Sciences and Intelligent Systems. His fields of interest are adaptive modeling of GMDH-type neural networks, fuzzy c-means clustering and classifier, data mining with fuzzy data analysis, human machine interface, and cognitive engineering.

E-mail: ichi@cs.osakafu-u.ac.jp 